\title{
Protée
}

\section{Quand la Bible s'ouvre à la lecture sémiotique}

\section{Jean-Yves Thériault}

Volume 34, numéro 1, printemps 2006

Fortune et actualité de $\mathrm{Du}$ sens

URI : https://id.erudit.org/iderudit/013311ar

DOI : https://doi.org/10.7202/013311ar

Aller au sommaire du numéro

Éditeur(s)

Département des arts et lettres - Université du Québec à Chicoutimi

\section{ISSN}

0300-3523 (imprimé)

1708-2307 (numérique)

Découvrir la revue

\section{Citer cet article}

Thériault, J.-Y. (2006). Quand la Bible s’ouvre à la lecture sémiotique. Protée, 34(1), 67-75. https://doi.org/10.7202/013311ar

\section{Résumé de l'article}

C'est au moment de la parution de Du sens que l'exégèse biblique et la sémiotique greimassienne se sont rencontrées. Après le choc initial et le dépassement des difficultés provoquées par le changement radical d'épistémologie, les disciplines ont su profiter l'une de l'autre. Au cours des années 1970, les études bibliques se sont tournées vers des approches synchroniques. D’autre part, la lecture de la Bible a contribué au développement de la sémiotique greimassienne : affinement de l'analyse des rapports entre sujets et développement de l'étude de la figurativité en direction de la mise en discours conçue comme productrice de signification. 


\title{
Q U AN D LA BIBLE S'OUVRE À LA LECTU RE SÉMIOTIQ U E
}

JEAN - Y VES THÉRIAULT

\begin{abstract}
Mais l'écriture sémiotique n'est pas seulement cette manifestation noble de l'histoire conçue comme une métamorphose des formes. Étant une praxis historique, elle ne peut faire autrement que manifester des contenus axiologiques et idéologiques, elle se dit même transformatrice de ces contenus, considérant leurs transformations comme le sens ultime de son faire. L'histoire jugera de l'efficacité de ces procédures.
\end{abstract}

(Greimas, 1970: 15)

\section{AU COMMENCEMENT!}

Le début de l'aventure biblique de la sémiotique greimassienne coïncide avec la publication du recueil d'articles intitulé $D u$ sens (1970). Se manifestait alors une crise des problématiques historiques dans les études bibliques. Se faisait sentir le besoin d'une réflexion fondamentale sur l'exégèse et ses méthodes dans le contexte du développement des études littéraires et des sciences du langage.

Ce n'est cependant pas la parution elle-même du livre qui a instauré la rencontre des disciplines. Comme amorce des rapports entre la sémiotique et les études bibliques, on doit rappeler l'importance d'une session de trois jours au Grand Séminaire de Versailles en septembre 1968: une trentaine de spécialistes de la Bible s'étaient réunis autour d'A.J. Greimas, reçu en tant que sémanticien susceptible d'intéresser les exégètes. Les exposés de quelques membres du séminaire de Greimas sur les textes bibliques étonnèrent les exégètes par leur manière d'aborder le texte "tel qu'en lui-même il se donne à lire dans la matérialité de son écriture» ${ }^{1}$. Le choc d'une théorie et d'une procédure d'analyse qui faisaient abstraction de la dimension historique et des conditions anciennes de production du texte bousculait des conceptions bien ancrées de la recherche biblique. D'autres rencontres eurent lieu ${ }^{2}$ et de nombreux biblistes furent ainsi sensibilisés à cette nouvelle approche critique des textes qu'on appelait encore «analyse structurale».

Après la sortie de Du sens de Greimas, le contact se poursuivit au gré de petits groupes de biblistes intéressés. Mettant en commun connaissances théoriques et tâtonnements sur les textes, l'analyse structurale entrait lentement dans le champ des 
études bibliques. On fit d'abord de l'analyse du récit. Trois courants étaient représentés: Bremond, Barthes et Greimas. Ce dernier paraissait plus difficile. Il demandait aux exégètes habitués aux méthodes historiques (recherche des sources et histoire de la formation des textes) une conversion radicale dans la manière de lire les textes et d'appréhender le sens: passer d'une approche diachronique à une lecture immanente et synchronique. L'influence de la sémiotique greimassienne sur l'exégèse biblique fut toutefois la plus stimulante du fait de son intérêt pour la sémantique. À partir de 1973, le séminaire de Greimas à Paris devint la principale source d'inspiration des biblistes intéressés par la sémiotique ${ }^{3}$.

\section{LE CHOC}

L'exégète adepte de l'approche historique disposait déjà d'un vaste ensemble de méthodes servant à reconstituer les milieux d'origine des textes, à retracer l'histoire de leur composition, à vérifier le sens des mots et la valeur historique des événements relatés dans la Bible. Plus que l'ajout de quelques techniques et procédés complexes à un arsenal interprétatif déjà impressionnant, la sémiotique exigeait un changement radical de point de vue sur les textes.

L'entrée de la sémiotique en exégèse biblique a marqué une rupture d'isotopie. Tradition-, Form-et Redaktionsgeschichte découlaient logiquement les unes des autres à l'intérieur $d u$ même paradigme. La méthode sémiotique relève d'un horizon épistémologique complètement étranger et importe dans le champ des études bibliques une attitude différente à l'égard du texte et une instrumentation sans lien avec celle de l'historico-critique.

(Genest, 1992: 11-21)

Habitués aux dissections en petites unités littéraires, aux strates de composition et à la recherche des sources, beaucoup de biblistes résistaient - et résistent encore - à cette approche qui s'intéresse au texte simplement comme il se donne à lire.

En effet, la visée originale de la sémiotique littéraire repose sur le croisement de deux axiomes structuraux: le principe d'immanence qui affirme l'existence d'un sens immanent à l'objet textuel étudié, ce sens devant être construit à partir des structures propres de cet objet; et le principe de la différence qui affirme que le sens est saisi dans l'appréhension des différences internes à l'objet. Ces deux principes vont à l'encontre des pratiques de la démarche historique alors dominante en exégèse: le recours explicatif par le contexte historique, l'histoire de la composition orientée vers l'intention de l'auteur et l'étude du vocabulaire par l'étymologie et l'intertextualité.

Il y eut aussi malentendu. On attendait de la sémiotique des résultats comparables à ceux des pratiques traditionnelles de l'exégèse, vérifiables et critiquables des points de vue habituels de la recherche biblique. On n'avait pas suffisamment saisi que la sémiotique n'était pas simplement une méthode à côté des autres pour l'interprétation des textes, mais qu'elle touchait à des questions plus fondamentales en recherche biblique, les «conditions premières de la saisie du sens - si l'on veut, de la production ou de la génération du sens -»(Greimas, 1970: 10); il fallait donc apprendre «à mieux connaître où il se manifeste et comment il se transforme» (ibid.: 17). Il fallait passer du sens conçu comme «fond», transmis par un auteur et interprété à partir de la "forme littéraire» lue en contexte de production historique, à la signification comme forme $d u$ contenu, un sens construit et articulé dans l'immanence du texte.

Plus concrètement, on reprochait à la sémiotique ses procédures d'analyse trop formelles, utilisant un vocabulaire bien compliqué. On trouvait l'investissement trop laborieux pour une récolte cognitive bien mince et une pâture théologique assez maigre. De fait, c'est la manière nouvelle de concevoir le texte et le sens qui faisait difficulté. Depuis un siècle, la Bible était considérée comme document historique source de révélation, réservoir de savoir et de message à interpréter et à transmettre fidèlement. On était maintenant appelé à la visiter comme monument littéraire, pour lire et entendre dans cette cathédrale langagière ce qui se dit du sujet humain comme être de langage et sujet de parole. 


\section{L'ANALYSE NARRATIVE}

L'introduction de la sémiotique dans le champ des études bibliques ouvrit cependant la lecture de la Bible aux nouvelles perspectives fournies par les sciences du langage et des systèmes signifiants. Au cours de la première décennie de pratique sémiotique pour la lecture des livres bibliques, c'est la grammaire narrative dans toute son extension qui fut mise à contribution. La syntaxe narrative fut présentée progressivement dans la revue Sémiotique et Bible, puis reprise plus systématiquement dans le manuel du Groupe d'Entrevernes (1979) ${ }^{4}$. Elle s'exerçait principalement sur la multitude des récits bibliques, en particulier les mini-récits des évangiles. On peut retenir comme ses meilleurs fruits les études de ce Groupe parues dans Signes et Paraboles (1977) 5 .

Les récits courts fournissaient un terrain privilégié d'apprentissage pour la mise en œuvre de la dimension narrative et de la dimension discursive alors organisée autour des rôles thématiques. Il était plus facile pour les exégètes, habitués à interpréter des mots et des phrases, de modifier leur pratique sur des récits, car ceux-ci étaient plus aisément reconnaissables comme unités construites selon des règles qui dépassent la structure de la phrase. Les schémas d'organisation narrative élaborés par Greimas se prêtaient bien à l'analyse des courts récits bibliques: on s'exerçait à identifier les programmes narratifs selon les quatre grandes phases et la triple épreuve, en essayant de préciser les rôles des actants et d'enregistrer l'inversion des contenus.

Au cours des années 1970, la pratique biblique de la sémiotique greimassienne reposait ainsi sur «Les jeux des contraintes sémiotiques», les "Éléments d'une grammaire narrative» et «La structure des actants du récit». Mais elle utilisait aussi les développements théoriques de la sémiotique $d u$ discours, élaborant la structure modale des sujets narratifs, l'articulation discursive du contenu et l'organisation de la dimension cognitive avec ses modalités véridictoires, épistémiques et aléthiques. Contenu théorique que nous retrouvons dans la table des matières du recueil Du sens II : «les objets de valeur»; «les actants, les acteurs et les figures»; «théorie des modalités"; "contrat de véridiction"; "le savoir et le croire». Tels sont les éléments théoriques et méthodologiques qui inspiraient la lecture sémiotique de l'écriture biblique, sur le modèle de l'«exercice pratique», réalisé par Greimas lui-même dans son Maupassant (1976), et des études présentées dans $D u$ sens II («La Ficelle» et «La soupe au pistou») ${ }^{6}$.

L'étude de la Bible profitait de la recherche sémiotique. Le repérage et l'articulation des fonctions, des rôles actantiels et des rôles thématiques ouvraient de nouvelles voies à la lecture des nombreux récits bibliques tout en servant à illustrer la grammaire narrative encore en élaboration. De plus, les exégètes furent sensibilisés aux catégories sémantiques fondamentales qui sous-tendent le discours dans les textes bibliques.

\section{LES DIFFICULTÉS}

Ces pratiques engendrèrent cependant une certaine dose d'insatisfaction, car la mise en ouvre d'un lourd appareillage théorique et méthodologique pour une analyse rigoureuse, qui aboutit simplement à une représentation assez formelle des textes, intéressait peu la recherche biblique. Ce que les lecteurs du texte sacré cherchent c'est le message, la parole, pas des modèles abstraits et des structures logiques, fussent-elles celles qui engendrent les textes à lire. On s'apercevait que les modèles sémiotiques ne donnaient pas la clé d'interprétation pour atteindre un «meilleur sens du texte", la formulation d'un message à cueillir comme fruit de la lecture. Une bonne partie des exégètes, qui avaient pris de la distance par rapport à l'étude diachronique et adopté une démarche plus synchronique, passèrent aux approches rhétoriques et narratologiques. Ces approches s'inspirent de modèles cherchant à rendre compte des stratégies communicatives, selon des procédures concevant le texte comme un médium chargé d'intentionnalité par une source émettrice.

C'est que tout en pratiquant la grammaire narrative, certains étaient restés dans une perspective de communication intentionnelle, en concevant un peu 
le sens comme le message qui passe de l'émetteur au récepteur, moyennant un encodage approprié; dans la ligne d'une sémiotique où le langage et les signes sont étudiés dans une perspective fonctionnaliste, c'est-àdire comme systèmes de communication (Prieto, Mounin, Martinet, Eco), où l'on s'intéresse à l'arrangement des signes pour une communication réussie, à la génération des messages entre les locuteurs, etc. La "composition rhétorique», lue comme visée persuasive ou argumentative, de même que l'«organisation narrative", reconnue comme disposition stratégique des faits et du savoir à l'adresse du lecteur, rattachent en effet le sens des textes à une source productrice s'exprimant dans une composition littéraire qui met en forme un message.

À notre avis, une dimension théorique de $D u$ sens et $D u$ sens II n'avait pas été suffisamment assimilée: la réflexion sur les enjeux sémantiques liés à la manière de considérer un texte. Il n'y avait pas eu de conversion appropriée dans la conception de ce qu'est un texte et des implications de son statut de manifestation d'un tout de signification. Il aurait fallu aussi mieux intégrer une donnée essentielle de la sémiotique greimassienne: le sens n'est pas donné directement par le signe, il n'est pas simplement un objet communicable selon un code approprié; il doit être conçu comme une saisie moyennant un réseau de relations qui sous-tend le système des signes, une architecture composée de structures relationnelles hiérarchisées. La signification est une affaire de différence, d'articulation et de mise en relation en attente d'un «lecteur» qui entre dans cette dynamique signifiante. Bien plus, les modèles proposés (carré sémiotique, schéma narratif, etc.) ne constituent ni une représentation ni une description du sens, ils ne sont que des instruments pour la saisie articulatoire du sens et de ses transpositions. Ils ne nous apprennent pas la nature du sens, mais ils nous aident à saisir les lieux de manifestation du sens et ses transformations (Greimas, 1970: 7-14).

Ne pas comprendre la signification comme articulation d'éléments de contenu dans une mise en discours a entraîné, entre autres, un mauvais usage du carré sémiotique, compris à tort comme une sorte de résumé de l'intrigue ou de synthèse du discours d'un texte. De plus, la représentation greimassienne de l'organisation du contenu, à la manière d'une articulation en trois paliers conçue comme un parcours génératif de la signification, semble faire disparaître tout "auteur" que les exégètes aiment concevoir comme source de l'écrit et responsable du discours. Bref, la difficulté souvent évoquée du langage sémiotique ("jargon compliqué», "vocabulaire abscons») cachait plutôt le difficile déplacement épistémologique d'une théorie de la signification. Il ne s'agissait pas simplement d'intégrer de nouvelles procédures d'analyse et d'interprétation, mais d'opérer un changement radical de conception du texte, du discours et de la saisie du sens.

\section{RELATION ENTRE DES SUJETS}

Reste que c'est cette sémiotique de la signification qui s'est avérée la plus féconde dans le domaine des études bibliques. Et là où elle a été pratiquée avec rigueur pour la lecture des textes bibliques, elle a contribué au développement de la recherche sémiotique elle-même. En effet, le travail de lecture des récits de miracles, des paraboles et des textes apocalyptiques, entre autres, a bien vite interrogé certains modèles théoriques (schéma narratif simple, passage du thématique au figuratif) et suscité quelques développements de la sémiotique standard, afin de mieux rendre compte des relations fiduciaires entre sujets et de la dimension figurative des textes.

L'Écriture sainte s'est vite mise à résister aux schémas narratifs simples et à se sentir à l'étroit dans la stricte modélisation en structures fondamentales. Les adeptes de l'approche sémiotique ont dû parfaire leurs outils d'analyse pour interpréter les récits bibliques où la subjectivité des acteurs s'entremêle avec la narration des actions ou des événements. Les récits de miracles s'intéressent moins au miracle comme tel qu'aux transformations des relations intersubjectives qui se tissent entre les acteurs - et à la nature de celles qui se jouent entre énonciateur et énonciataire.

Il arrive que les récits bibliques relativisent la réalisation d'un 
désir (de guérison, par exemple) au profit de la relation qui s'établit entre l'acteur de la quête et celui de l'exaucement: le récit fait sens davantage de leur approche, de leur dialogue, de leur accord ou désaccord, que de la transformation corporelle désirée et réalisée. Si bien que dans l'exaucement, le bien communiqué n'est plus ce qui vient simplement combler le manque, il couronne un itinéraire de reconnaissance où le croire et l'écoute de la parole sont mis à l'épreuve. Il devient le signe corporel qui renvoie à un autre type d'incomplétude.

(Delorme, 1992a: col. 320-321)

Dans certains récits de guérison, la guérison ne constitue pas la transformation principale, car les parcours figuratifs construisent d'autres valeurs liées à l'interrelation des sujets. Dans les évangiles, quand des débats s'élèvent autour d'une guérison ou d'un exorcisme, on se rend compte que l'objet de valeur dépend des rapports qui se nouent entre des sujets inscrits dans des parcours figuratifs - et des prises de parole - qui doivent être finement analysés. Dans la constitution du sujet, la quête de l'objet se trouve atténuée au profit des enjeux d'une rencontre de sujets dans la parole. La pratique de la lecture des textes bibliques faisait ainsi constater que la grammaire narrative, telle qu'elle était alors comprise, ne suffisait pas pour rendre compte des virtualités signifiantes qui se manifestaient dans la représentation figurative des acteurs et dans l'intrication inattendue de divers champs sémantiques (dans les textes bibliques lus, le thématique paraissait incohérent). Ces difficultés de lecture appelaient le développement de procédures d'analyse plus attentives au dispositif figuratif, tel qu'il est organisé dans la mise en discours particulière du texte lu? .

De même, les paraboles évangéliques devinrent des lieux privilégiés d'analyse et de mise à l'épreuve des ressources de la sémiotique greimassienne ${ }^{8}$. Récits métaphoriques enchâssés dans un grand récit, elles représentaient un défi particulier pour les outils habituels de l'analyse narrative et pour l'observation de la transformation des valeurs sémantiques. Elles ont entraîné certains ajustements des modèles fondamentaux et une évolution des stratégies d'analyse. D'une part, l'interaction originale de deux plans de signification, le récit parabolique et le récit englobant, fournissait aux sémioticiens un phénomène typique du traitement des figures par le discours. D'autre part, le langage parabolique s'avérait un terrain favorable pour le développement de l'opposition entre un savoir par démonstration et un savoir qui procède plutôt par analogie ou par enchainements figuratifs; et de l'opposition entre une pensée logique et une pensée qui s'adresse davantage au croire, à la fiducie nécessaire à toute relation entre les humains.

Les réflexions de Greimas sur «Le savoir et le croire» comme "un seul univers cognitif» (Du sens II: 115-134) sont en lien avec ce travail de lecture des paraboles. Dans ce chapitre qui vise à mieux reconnaître la part du fiduciaire et du logique dans la constitution du savoir, Greimas évoque en effet le «fonctionnement discursif de l'allégorie et de la parabole» pour indiquer l'aptitude du discours figuratif

[...] à projeter une double référence, la première en profondeur et créatrice d'une isotopie thématique plus abstraite, et la seconde, en latéralité, développant une nouvelle isotopie figurative parallèle. (Ibid.: 131)

\section{LA FIGURATIVITÉ ET SES «CAPACITÉS» SIGNIFIANTES}

Dans l'élaboration du parcours génératif de la signification, Greimas avait établi une composante discursive comportant un plan syntaxique, l'organisation des grandeurs figuratives autour des trois axes acteurs, temps, espace -, et un plan sémantique, celui des valeurs thématiques assumées concrètement par les figures. La figurativité particulière des textes de la Bible a provoqué une élaboration plus précise de la dimension figurative, une mise à l'épreuve de ses rapports avec la composante narrative et une meilleure reconnaissance de son apport dans l'articulation signifiante du fait de sa mise en discours. On a été ainsi amené à reconnaître que le plan discursif présente une réelle autonomie: le dispositif figuratif n'est pas seulement la concrétisation ou l'«habillage» de valeurs thématiques abstraites, mais il produit une articulation du sens dans le discours. 
Postulant que la signification s'articule dans la saisie de la différence, Greimas en avait explicité les conditions en élaborant le modèle caractéristique appelé parcours génératif de la signification. Dans ce parcours qui va de la structuration élémentaire du sens (représentée par le carré sémiotique) à sa manifestation textuelle, Greimas utilise le terme conversion quand il s'agit de l'opération permettant de passer du niveau des structures profondes au palier narratif: les relations et les opérations représentées par le carré se trouvent converties en sémantique et syntaxe narratives, formant des programmes narratifs. Mais, quand intervient le plan discursif où les structures sémio-narratives sont prises en charge par la composante discursive, le terme conversion s'avère moins approprié pour décrire le passage d'un niveau à l'autre; c'est alors le vocable convocation qui rend compte du processus d'articulation des figures dans le réseau signifiant.

Selon Louis Panier, ce changement dans le vocabulaire est le signe en fait d'une difficulté sérieuse dans la trajectoire du parcours génératif:

En effet, la convocation des grandeurs figuratives (effet de la praxis énonciative) est une opération complètement différente de la conversion du niveau élémentaire au niveau narratif. Il devient alors difficile de s'en tenir à une vision purement générativiste du parcours sémiotique. (2001: 66, note 15)

De fait, l'opération de convocation appelle une instance d'énonciation pour sa mise en œuvre et le terme convocation (vocare) évoque une voix qui appelle, une instance de parole. C'est en ce lieu de rencontre et de tension, entre ce qui est généré par les structures profondes et la mise en dispositif figuratif, que s'inscrit une instance énonciative productive dans la mise en œuvre du sens. Cette instance énonciative ne se réduit pas à un mécanisme de transformation de structures; s'y réalise la mise en discours de l'ensemble signifiant manifesté dans un texte. Le lieu d'achoppement du parcours génératif devient le lieu du développement théorique élaboré par le CADIR 9 .

Dans l'examen approfondi de la mise en discours, l'énonciation ne doit pas être mise entre parenthèses; elle s'avère plutôt un lieu majeur où la signification s'inscrit et se développe à l'intérieur même du texte. Mais cette prise de distance, par rapport à l'une des articulations du modèle génératif, n'est pas un abandon de la sémiotique greimassienne. Cette sémiotique postule que les éléments signifiants reçoivent leur signification de leur insertion dans le réseau discursif. L'instance discursive, en associant et en opposant des signes, se trouve à les décomposer en unités minimales, à les articuler selon de nouveaux réseaux, constituant ainsi des significations qui ne sont plus celles du lexique. Lire, c'est donc construire ce réseau de traits distinctifs pour former un ensemble signifiant (la forme du contenu), c'est se mettre en quête de sens en suivant l'organisation interne des éléments sémantiques. Certes, les mots gardent mémoire de leurs usages antérieurs, mais leur signification dans un texte relève essentiellement de la structure du discours qui les porte, ce texte étant considéré comme un tout où les composantes linguistiques et littéraires se fondent en une cohérence signifiante originale. L'architecture interne du texte n'est pas reçue comme expression d'un auteur, mais elle est reconnue comme manifestation d'une unité signifiante à construire dans un travail de lecture qui prend en considération l'articulation interne de toutes les unités et de tous les niveaux de signification. Une manière donc de reconnaître la signification dans la combinaison des éléments au niveau du discours conçu comme organisation signifiante du langage.

La lecture sémiotique des textes bibliques poussée jusque dans leurs résistances a amené, en effet, à reconnaître que le dispositif figuratif ne se contente pas de manifester du thématique: il renvoie à une instance énonciative où s'élabore un discours fait de l'articulation particulière de ce réseau signifiant unique. Ce niveau, où l'on analyse le jeu des figures représentées par des acteurs en interrelation dans l'espace et le temps, ne se borne pas à la manifestation des structures profondes. Il relève davantage d'une instance d'énonciation qui contribue à l'élaboration du sens par l'agencement de ces parcours figuratifs dans cet ensemble signifiant. La lecture n'est donc pas un simple décodage des figures, mais une activité 
attentive au dynamisme de la mise en discours. Le travail d'énonciation (assumé par le lecteur) transforme le contenu des figures et construit la signification dans le dispositif figuratif particulier qui est donné à lire. Le sens s'élabore dans la mise en discours relevant de l'énonciation. Autrement dit, l'œuvre de mise en discours doit être considérée comme une organisation fondatrice de signification.

\section{L'ÉNONCIATION IMPLIQUÉE PAR LA MISE EN DISCOURS}

L'analyse des prises de parole et des dispositifs figuratifs entrelacés dans un texte conduit à prendre en considération l'énonciation qu'ils attestent, l'instance qui fait tenir ensemble tous les parcours discursifs et narratifs. On doit compter avec les écarts et les rapports nouveaux engendrés par l'activité de mise en discours considérée selon ses deux pôles: énonciateur et énonciataire. L'énonciation crée des enchaînements et des associations qui travaillent le sens et génère de nouvelles significations.

L'articulation singulière de chaque texte provoque une construction du sens, élabore un discours qui est à retracer justement dans cette nouvelle organisation discursive particulière.

Au CADIR de Lyon ${ }^{10}$, on a ainsi développé une stimulante réflexion sur le statut sémiotique des grandeurs figuratives dans les textes et sur leur place dans le dispositif de l'énonciation. Certes, la figure est reconnue comme un élément de contenu ayant valeur de représentation, une correspondance avec un élément du monde naturel. Et, quand les figures sont mises en parcours dans un texte, elles s'investissent de valeurs thématiques du fait de l'isotopie discursive. Ce niveau thématique ne marque pas toutefois la fin de l'opération figurative.

Mais du fait de leur mise en discours dans un texte particulier, ces éléments figuratifs acquièrent un statut discursif, ils ne sont plus là seulement pour forger l'impression référentielle du texte en représentant des états de choses, ils ne sont pas là seulement comme concrétisation de valeurs thématiques abstraites, ils entrent dans les formes figuratives de la mise en discours et deviennent par là les formants d'une structure de la signification. (Panier, 2001: 64) ${ }^{11}$
Quand elle est mise en discours, vient un moment où la figure se vide de la signification de sa configuration virtuelle pour pouvoir entrer dans le dispositif figuratif spécifique où elle est inscrite. Certes, cette opération de suspens du sens garde quelque chose d'une capacité figurative minimale (le figural ${ }^{12}$ ), un support minimal d'investissement sémantique, mais elle met aussi en opération discursive et en quête de signification un sujet d'énonciation. Souvent, la difficulté ou même l'impossibilité d'organiser de façon cohérente ou satisfaisante le thématique signale cette opération signifiante relevant de l'énonciation.

Les figures en discours sont ainsi le lieu d'une tension entre représentation, signification et instauration d'un sujet structuré par la langue. L'instance de l'énonciation, présupposée par le discours, s'atteste dans la convocation, la sélection et l'agencement des figures de contenu en un tout de signification. Nous avons ainsi appris à compter avec cette instance de mise en discours qui «habite en quelque sorte le discours et le rend lisible, parlant» (Delorme, 1992b: 7). Selon Louis Panier, la sémiotique interprétative pratiquée au CADIR s'intéresse moins à la fonction figurative qu'à

[...] l'opérativité sémantique et énonciative $d u$ figuratif en concevant la figure comme une grandeur instaurée lors de la mise en discours et donc corrélative d'émergence d'une instance d'énonciation. (2004: 41)

\section{Et J. Delorme écrit:}

Il devient possible, sans céder à l'imaginaire, de lire le texte comme la trace d'un sujet parlant et de se disposer au passage d'une parole qui provoque le lecteur à interpréter. (1993: 40)

\section{DE RETOUR À DU SENS}

Nous paraissons loin de la problématique examinée dans le recueil Du sens. Pourtant, ce développement de la compréhension de la figurativité et de la mise en discours dans l'énonciation repose sur des observations et des orientations déjà présentes dans cet ouvrage.

L'affirmation de l'arbitraire du signe, tout en permettant des

progrès considérables dans la connaissance de la structure interne des langues dites naturelles, n'a pas manqué d'élargir [...] la 
problématique du statut du langage [...]. Si le rapport entre le signifiant et le signifié, au niveau du signe, c'est-à-dire $d u$ mot ou d'une unité syntagmatique quelconque, est arbitraire, il l'est aussi au niveau de tous les discours par lesquels la langue est présente à nous [...]. (Greimas, 1970: 49)

Le développement de la théorie de la figurativité présenté dans cet article constitue une prise en compte de cette "affirmation de l'arbitraire du signe" poussée jusque dans le dispositif figuratif et la mise en discours. Dans l'agencement des parcours figuratifs, les figures (signifiants) sont vidées de leur contenu (signifié) et elles deviennent disponibles pour d'autres investissements sémantiques issus de la mise en discours particulière qui les agence.

Et plus loin, dans ce même chapitre sur «Les conditions d'une sémiotique du monde naturel», nous lisons:

Un problème théorique subsiste néanmoins: c'est celui de la désémantisation, toujours possible, des éléments constitutifs des énoncés gestuels, que nous avons évoqué précédemment.

Ainsi, on peut se demander, à propos de la gestualité qui se veut esthétique, dans le ballet par exemple - et tout en reconnaissant sa nature de code artificiel, puisque composite, comportant, entre autres, des séquences mimétiques évidentes, et surtout puisque le spectacle n'est dans sa totalité qu'un énoncé produit par ce sujet de l'énonciation, le chorégraphe -, si la "désacralisation" du discours mythique n'a pas entraîné la désémantisation des énoncés gestuels, ne laissant à la gestualité esthétique, pour signifier, que les formes narratives $d u$ discours. (Ibid.: 81; nous soulignons)

Certes, le domaine est différent (de la langue à celui de la danse) et la perspective prise par Greimas est plutôt celle de la communication, mais l'affirmation de la désémantisation des éléments constitutifs, du fait de leur inscription dans une totalité produite par un sujet de l'énonciation, est là comme germe montrant sa fécondité dans l'exploration de la figurativité en direction du sujet d'énonciation. Autrement dit, les figures se vident de leur contenu pour former de nouveaux signifiants dans le réseau énonciatif qui les prend en charge, comme les éléments gestuels dans une chorégraphie. L'orientation prise au CADIR a contribué à développer une compréhension discursive et interprétative de ces réflexions générales de Greimas sur l'énonciation.

Ces dernières remarques voulaient simplement souligner la souplesse de la sémiotique élaborée dans $D u$ sens et $\mathrm{Du}$ sens II. C'est ainsi que Louis Panier peut donner le titre «Les sémiotiques d'A.J. Greimas» à un article dans lequel il montre

[...] comment le projet scientifique inauguré par Greimas s'est trouvé en constant renouvellement à partir des découvertes que son évolution même soumettait à la réflexion. La sémiotique greimassienne, loin d'être un corps figé de doctrine comme on a souvent voulu le croire, est un projet, une dynamique de la recherche. (2001: 55)

Le pluriel, «Les sémiotiques», souligne les possibilités d'évolution et de transformation de ce "projet»: c'est un dispositif théorique vaste et riche qui mérite encore exploration et approfondissement. Nous en avons montré quelques fruits dans la sémiotique interprétative des textes bibliques. Le plus grand apport de cette sémiotique dans le domaine biblique fut l'apprentissage d'une lecture attentive à ses procédures, non pour dire le sens avec plus de compétence, mais pour instaurer le texte comme discours et, en définitive, comme parole de l'Autre. 


\section{N O TES}

1. J. Delorme, 1993: 38. Du même auteur, voir l'article

encyclopédique «Sémiotique», 1992a. Plus récemment, voir Delorme, 2001. Notre présentation s'appuie largement sur ces travaux.

2. Notamment le Congrès de l'A.C.F.E.B. à Chantilly, en septembre 1969. La conférence de R. Barthes, de même que les travaux des

ateliers et de la table ronde qui ont suivi ont été publiés dans Exégèse et Herméneutique, 1971. Voir aussi R. Barthes, F. Bovon et alii, 1971. Pour l'évolution de la sémiotique greimassienne en milieu biblique américain, que nous connaissons moins, voir D. et A. Patte, 1978; D. Patte, 1989 et 1990.

3. On trouve, dans l'article de J. Delorme (1992a), la mention des centres et des groupes qui se sont formés ainsi qu'une abondante bibliographie. Pour une présentation plus détaillée de ces débuts, voir aussi J. Delorme, 2001.

4. Cet excellent guide correspond à la réflexion et à la pratique sémiotique à la fin des années 1970. Plus récentes, la présentation de J.-C. Giroud et L. Panier (1987) et l'initiation pratique de W. Vogels (1988) ont aussi servi de manuel aux biblistes intéressés à la sémiotique. 5. Notons aussi les lectures faites par les membres du groupe ASTER d'un certain nombre de récits évangéliques qui mettent en scène des femmes (1987). ASTER (Atelier de sémiotique du texte religieux) est un groupe interuniversitaire (au Québec et à Ottawa) qui pratique la sémiotique greimassienne.

6. Les principales revues faisant état de l'approche sémiotique des textes bibliques sont: Sémiotique et Bible (Lyon); Foi et Vie. Cahiers Bibliques (Paris); Linguistica Biblica (Bonn); Semeia (États-Unis). Pour l'énumération des divers travaux effectués sur les textes bibliques, voir les articles de J. Delorme.

7. Il faut noter alors l'influence de J. Geninasca (1977 et 1990).

8. Les diverses formes de discours paraboliques constituent un champ que la sémiotique a volontiers exploré: voir Groupe d'Entrevernes, 1977 ; J. Delorme, 1987 et 1989.

9. Centre pour l'Analyse du discours religieux, rattaché à la Faculté de théologie de l'Université catholique de Lyon.

10. Nous pensons en particulier aux divers travaux de J. Delorme, J. Calloud, L. Panier et F. Martin qu'on trouve énumérés dans les références bibliographiques de J. Delorme (2001).

11. Voir aussi Panier (1993 et 1995).

12. Le terme figural vient du sémioticien J. Geninasca. Il sert à définir le statut des figures comme éléments signifiants, dont le signifié est déterminé par leur mise en discours. Un peu comme le visuel par rapport au visible dans un tableau non figuratif.

\section{RÉFÉREN CES BIBLIO G RAPHIQ U ES}

ASTER [1987]: De Jésus et des femmes. Lectures sémiotiques, Montréal et Paris, Bellarmin et Cerf;

[2005]: Le Déluge et ses récits : points de vue sémiotiques, Québec,

Presses de l'Université Laval.

BARTHes, R. (dir.) [1971] : Exégèse et Herméneutique, Paris, Seuil.

BARTHES, R., F. BOVON et alii [1971]: Analyse structurale et exégèse

biblique, Neuchâtel, Delachaux.

Delorme, J. [1987]: Parole. Figure. Parabole, Lyon, Presses universitaires de Lyon;

_ (dir.) [1989]: Les Paraboles évangéliques. Perspectives nouvelles, Paris,

Cerf ;

__ [1992a]: Article "Sémiotique", Dictionnaire de la Bible.

Supplément, Paris, Letouzey \& Ané, fascicule 67, col. 281-333;

12

[1993]: "La sémiotique greimassienne et les études bibliques ",

Nouveaux Actes Sémiotiques, no 25, 35-44;

_ [2001]: "La sémiotique littéraire interrogée par la Bible",

Sémiotique et Bible, no 102, 3-28 et n 103, 3-21 (éd. originale:

"Orientations of a Semiotics Questioned by the Bibel ", Semeia, n 81, 1998, 27-62);

GENEST, O. [1992]: «Analyse structurale et exégèse biblique», dans

R. Latourelle (dir.), Dictionnaire de théologie fondamentale, Montréal et

Paris. Bellarmin et Cerf, 11-21.

GeninASCA, J. [1977]: «Pêcher/Prêcher. Récit et métaphore (Luc 5, 1 -

11)», dans Groupe d'Entrevernes (1977), 144-173;

[1990]: "Du texte au discours littéraire et à son sujet», Nouveaux

Actes Sémiotiques, nos 10-11, 9-34.

GIROUD, J.-C. et L. PANIER (dir.) [1987]: "Sémiotique. Une pratique de lecture et d'analyse des textes bibliques", Cahiers Évangile, no 59, 5-52.

Greimas, A. J. [1970]: Du sens. Essais sémiotiques, Paris, Seuil;

[1976]: Maupassant. La sémiotique du texte. Exercices pratiques,

Paris, Seuil;

[1983]: Du sens II. Essais sémiotiques, Paris, Seuil.

Groupe D’EnTREVERnEs [1977]: Signes et Paraboles. Sémiotique et texte évangélique, Paris, Seuil;

- [1979]: Analyse sémiotique des textes. Introduction. Théorie. Pratique, Lyon, Presses universitaires de Lyon.

PANIER, L. [1993] : "Le statut discursif des figures et l'énonciation ", Sémiotique et Bible, no 70, 13-24;

[1995]: «Devenir des figures, figures en devenir. La théorie des figures dans l'exégèse biblique ancienne», dans J. Fontanille (dir.), Le Devenir, Limoges, Presses universitaires de Limoges, 147-157 (repris en 2000 dans Sémiotique et Bible, no 100, 14-24);

[2001]: «Les sémiotiques d'A. J. Greimas", Sémiotique et Bible, $\mathrm{n}^{\circ} 104,55-67$;

[2004]: "Figurativité, mise en discours, corps du sujet",

Sémiotique et Bible, no 114, 39-52.

PATTE, D. [1989]: Greimas's Structural Semiotics and Biblical Exegesis,

Atlanta, Scholars Press;

[1990]: Structural Exegesis for the New Testament Critics,

Minneapolis, Fortress Press.

Patte, D. et A. [1978]: Pour une exégèse structurale, Paris, Seuil.

VOGELS, W. [1988]: La Bible entre nos mains. Une initiation à la

sémiotique, Montréal, Ed. Paulines et SOCABI. 